\title{
Reproducibility of postprandial glucose responses to identical meals
}

\author{
A.M. Curran, K. Horner and L. Brennan \\ Institute of Food and Health, School of Agriculture and Food Science, University College Dublin, Dublin 4, Ireland
}

The prevalence of type 2 diabetes and the metabolic syndrome has increased in recent years. Maintenance of glucose levels within the normal range is crucial to prevent and decrease the risk of such diseases. Challenging homeostasis is key determinant of assessing a person's disease risk. Previous research has uncovered high variability in the response of different people to the same food, and have discovered high reproducibility of the same person's response to identical meals ${ }^{(1)}$. Differences in postprandial glucose responses highlight the need for personalised nutrition, in contrast to the 'one size fits all' approach to dietary interventions ${ }^{(2)}$. The objective of this study was to assess the reproducibility of the postprandial glucose response to a study breakfast and study evening meal for 9 days using a novel flash glucose monitoring system.

The cohort consists of 8 overweight or obese individuals between the ages of 40-65 years, free of prescription medication. Each participant was provided with 9 study breakfasts and 9 study evening meals to consume in a free living setting. Study meals comprised of approximately $75 \mathrm{~g}$ of carbohydrate. Postprandial glucose responses were captured using the FreeStyle Libre glucose monitor, which provides us with interstitial glucose readings every 15 minutes. Intra and inter-reproducibility of postprandial glucose response to study meals were calculated. This study was performed according to the Declaration of Helsinki.

High intraclass correlation co-efficients (ICCs) were obtained on an individual level over the 9 day period between total area under curves for glucose levels obtained from study breakfasts (ICC 0.867 (95\% confidence interval (CI) 0.669-0.982) with an average within person co-efficient of variation (CV) of $7.2 \%$ (SD $2.6 \%)$ ). A similar but slightly lower trend for study evening meals emerged (ICC $0.622(95 \%$ CI $0.313-0.936)$ with an average within person CV of $12.7 \%$ (SD $7.7 \%)$ ). Increased variability was observed when different participants consumed an identical meal. The inter-individual co-efficient of variations (CVs) were higher, with an average inter-individual CV of $22.2 \%$ (SD $4.1 \%$ ) for the study breakfast and a CV of $19.1 \%$ (SD $6.3 \%$ ) for the study evening meal, displaying increased variability in glucose response between individuals.

The results demonstrated high reproducibility of a person's response to the same food on an individual level and an increased variability in response between individuals. Diet plays a huge role in determining glucose levels, with postprandial glucose responses aiding in determining an individual's risk for the development of type 2 diabetes. Future research into the determination of underlying mechanisms leading to the variation in postprandial glucose responses will allow for unique opportunities in personalised nutrition.

1. Zeevi D, Korem T, Zmora N et al. (2015) Cell 163(5), 1079-1094.

2. Matthan NR, Ausman LM, Meng H et al. (2016) Am J Clin Nutr 104 (4), 1004-1013. 